\title{
The use of prebiotics and probiotics in infant formula
}

\author{
Ackerberg TS, BScDietetics; Labuschagne IL, BScDietetics; Lombard MJ, PhD \\ Nutrition Information Centre, Stellenbosch University \\ Correspondence to: Martani Lombard, e-mail: martani@sun.ac.za, nicus@sun.ac.za \\ Keywords: infant formula, prebiotics, probiotics, infant nutrition, feeding
}

\begin{abstract}
Gastrointestinal flora influences health, but the composition of flora can be changed with prebiotics or probiotics. The addition of probiotics to powdered infant formula has not been demonstrated to be harmful to healthy term infants. However, evidence of clinical efficacy regarding their addition is insufficient to recommend the routine use of such formula. The administration of probiotic (single or in combination) supplementation in infant or follow-on formula, and given beyond early infancy, may be associated with some clinical benefits, such as a reduction in the risk of nonspecific gastrointestinal infections, a reduced risk of antibiotic use and a lower frequency of colic and irritability. Confirmatory well-designed clinical research studies are necessary.
\end{abstract}

\section{Introduction}

Gastrointestinal flora influence health, ${ }^{1}$ but the composition of flora can be adapted by consuming prebiotics or probiotics. ${ }^{2}$ Prebiotics are nondigestible substances that stimulate the growth of health-promoting bacteria in the colon, such as bifidobacteria. ${ }^{3-5}$ They are live microbial feed supplements which improve the intestinal microbial balance. $^{4}$

Immediately after birth, bacterial colonisation of the infant's gut begins with bifidobacteria and lactobacilli. ${ }^{6}$ These organisms are transferred from the maternal microbial flora in the genital tract and colon during delivery, and also from the environment. ${ }^{4}$

Gut-associated immune tissue comprise $80 \%$ of the immune system, making the composition of intestinal flora an important factor in the immune system. ${ }^{7}$ It is believed that the onset of many diseases possibly relates to disruption of the early colonisation of the gut. ${ }^{5}$ Preterm infants have delayed colonisation of the gut with beneficial flora by three to four weeks, but colonisation with pathogens occurs earlier and contributes to health problems, such as necrotising enterocolitis (NEC). ${ }^{8}$

Breastfed infants are often healthier than formula-fed infants and can fight infections better. ${ }^{9}$ Breast milk naturally contains prebiotics (oligosaccharides) at a level of 10-12 g/l. These oligosaccharides favour the growth of bifidobacteria in the colon. Exclusively breastfed infants have higher numbers of bifidobacteria and lower numbers of the Escherichia coli bacteria, while formula-fed infants harbour equal amounts of these different types of intestinal flora. $3 ., 6,10,11$
There are various reasons for this, including the lower content and different composition of proteins in breast milk, the lower phosphorous content, and oligosaccharides and mediators of immune function that are found in human milk. Infant formula lacks these benefits. ${ }^{1}$ Breastfeeding protects against allergies and infections. This is thought to be partly due to the presence of more bifidobacteria in the gut. Therefore, breast milk stimulates the development of the infant's immune system. ${ }^{2}$ Bifidus-dominated flora is protective as it activates the immune system and inhibits invading pathogens that can cause infections. There is also some evidence that infants who suffer from allergies have less bifidobacteria and lactobacilli in their colons. ${ }^{2}$ Often, infant formula is supplemented with probiotics and prebiotics to help promote the development of a bifidusdominated flora, with the goal of creating an intestinal flora composition that is similar to that of a breastfed infant. ${ }^{3}$

\section{Probiotic-supplemented formula}

The overall health benefit and efficacy of adding probiotics to infant formula remains to be demonstrated in large randomised clinical trials (RCTs). A clinical report by the American Academy of Paediatrics reviewed the currently known health benefits of probiotic and prebiotic products, including those that are added to commercially available infant formula and other food products for children. ${ }^{5}$

The report states that the use of probiotics has been shown to be modestly effective in RCTs in treating acute viral gastroenteritis in healthy children, and preventing antibiotic-associated diarrhoea in healthy children. There is some evidence that probiotics prevent NEC in very low birthweight infants (birthweight between 1000-1 $500 \mathrm{~g}$ ), but more studies are needed. 
The committee on nutrition of the European Society for Paediatric Gastroenterology, Hepatology and Nutrition (ESPGHAN) systematically reviewed published evidence relating to the safety and health effects of the administration of formula that was supplemented with probiotics and/or prebiotics, and compared it to that on unsupplemented formula. $^{12}$

The committee concluded that there currently are no safety concerns regarding feeding probiotic- and/or prebioticsupplemented formula to healthy infants, but there are insufficient data to recommend the routine use of probioticand/or prebiotic-supplemented formula. They acknowledge the importance of more research in this field.

An effective probiotic must be nonpathogenic and nontoxic and exert a beneficial effect on the host. Moreover, it should be capable of surviving passage through the gastrointestinal tract, particularly the harsh environmental conditions in the human stomach and small intestine. Probiotic supplementation in infant formula has shown that some strains may persist in the infant gut and lower stool $\mathrm{pH}^{4}$

\section{Probiotics in the treatment or prevention of selected clinical diseases}

\section{Infectious diarrhoea}

Results of published RCTs have indicated that there is modest benefit when giving probiotics to prevent acute gastrointestinal tract infections in healthy infants and children. The strains of probiotics that were used included Lactobacillus rhamnosus GG (LGG), Streptococcus thermophilus, L. casei, Bifidobacterium lactis, or L. reuteri mixed with milk or infant formula or given as an oral supplement. Rotavirus was the most common cause of acute diarrhoea in the RCTs. ${ }^{5}$

Results of meta-analyse ${ }^{13-15}$ and a Cochrane review ${ }^{16}$ have been published on the benefit of probiotics in treating acute infectious diarrhoea in children. These reports indicate that probiotics reduce the number of diarrheal stools and the duration (approximately by one day) of the diarrhoea.

\section{Antibiotic-associated diarrhoea}

Reviews showed that use of a probiotic-supplemented formula reduces the incidence of antibiotic-associated diarrhoea. $B$. lactis and $S$. thermophilus had the greatest effect. ${ }^{5,7}$ A meta-analysis ${ }^{17}$ of published results from RCTs on probiotic use in the prevention of antibiotic-associated diarrhoea in children indicated a beneficial effect. Children received either a probiotic-supplemented formula or a separate probiotic as preventive treatment. No RCTs have been published that have investigated the effect of probiotics when treating antibiotic-associated diarrhoea in children. Thus, their use cannot be recommended. ${ }^{5}$

\section{Infantile colic}

Formula with B. lactis BL999 and LPR, L. reuteri or LGG is also not associated with a decreased frequency of colic, crying and irritability in children younger than six months, ${ }^{12}$ but the use of $B$. lactis and $S$ thermophilus is associated with a lesser frequency of colic in children older than six months. No effect was observed with the use of $B$ lactis alone, $L$. reuteri or L. salivarius. ${ }^{12}$

It is speculated that probiotics may change patterns of fermentation in the colon, leading to less gas or water fermentation, which may improve gastrointestinal tolerance. $^{10}$ Further confirmatory RCTs are required to recommend the routine use of probiotics in the treatment of infantile colic in both breastfed and formula-fed infants. ${ }^{5}$

\section{Respiratory infections}

Limited evidence shows that formula that is supplemented with $B$. lactis does not decrease the risk of respiratory infections, and $B$. longhum BL999 and L. rhamnosus LPR are not associated with a decreased use of antibiotics in infants younger than six months. ${ }^{12}$ There is also limited evidence to show that a combination of $B$. lactis and $S$. thermophilus or $L$. reuteri is associated with a decreased use of antibiotics in children older than six months. ${ }^{12}$

\section{Allergies}

Insufficient evidence was found to recommend the use of probiotics in infant feeds to prevent food allergies or sensitivities., 5 The ESPGHAN concluded that limited available data suggest that the probiotics studied (B. longhum BL999 and LPR) had no effect on allergies. However, the committee considered that there is too much uncertainty to draw reliable conclusions from the available data. $^{12}$

\section{Prebiotic-supplemented formula}

Examples of prebiotic oligosaccharides include fructooligosaccharides (FOS), inulin, galacto-oligosaccharides (GOS), and soybean oligosaccharides. ${ }^{5}$ Supplemented bifidobacterial species colonise the intestine of infants who receive the supplementation. ${ }^{10}$ The use of oligosaccharides in formula results in higher bifidobacteria counts in stools. Faecal lactobacilli can also increase, but the clinical benefits are not clear. ${ }^{6,12}$ Prebiotic-supplemented formula has a limited effect on the reduction of pathogenic bacteria. The response to prebiotics in formula is thought to be dependent on the healthy gut flora prior to supplementation. Some studies show a dose-dependent effect of the prebiotics on the stimulating effect of bifidobacteria and lactobacilli growth. ${ }^{6}$

Supplementation of formula with a mixture of FOS/GOS has no negative effects. There is a modest improved effect on growth in children younger than six months. ${ }^{12}$ However, it is associated with a slightly higher weight gain in children older than six months. ${ }^{6}$

The use of prebiotics in formula can potentially reduce the stool $\mathrm{pH}$ of children younger than six months. ${ }^{6,12}$ Limited evidence shows that such formula potentially leads to increased stool frequency and softer stools, similar to that of breastfed infants. 6,12 
Furthermore, infants who receive GOS/FOS might have fewer episodes of infections and require fewer antibiotics. The total number of infections, cumulative incidence of infections and the number of recurring infections during the first six months might be reduced. ${ }^{11}$ However, evidence regarding the use of prebiotics in the prevention of allergies is inconclusive. ${ }^{19}$

Prebiotic-supplemented infant formula is mostly well tolerated. To date, these products seem to be safe in healthy infants. FOS and GOS may be voluntarily added to infant formula $(<0.8 \mathrm{~g} / 100 \mathrm{ml})$ in a ratio of $90 \%$ GOS: $10 \%$ FOS. The Food and Agricultural Organization (FAO) of the United Nations supports the supplementation of formula with prebiotics in infants aged five months and older, as these infants will have a mature immune system and intestinal colonisation.

\section{Prebiotic- and probiotic-supplemented formula in preterm neonates}

There is limited evidence that the supplementation of preterm formula with FOS/GOS is well tolerated, increases the bifidobacteria stool colony counts, decreases the growth of pathogenic bacteria, improves gastrointestinal transit time, and softens and acidifies stools to a degree that is similar to that in breastfed infants. Therefore, supplementation with prebiotics is safe, but routine use is not recommended. ${ }^{8}$ Premature infants have inadequate colonisation of the gut for various reasons. It is suspected that the establishment and composition of intestinal flora in preterm infants plays a role in the development of NEC. Theoretically, administration of probiotics to preterm infants should reduce gut pathogens, improve the structure and function of the gut, reduce the need for parenteral nutrition, facilitate enteral nutrition, improve the gut mucosal barrier function, decrease sepsis and antibiotic use, and prevent NEC.

\section{Conclusion and recommendations}

The prebiotics and probiotics that are now being added to commercial infant formula are classified as GRAS (generally regarded as safe) by the FDA.

The addition of probiotics to powdered infant formula has not been demonstrated to be harmful to healthy term infants. However, evidence of clinical efficacy regarding their addition is insufficient to recommend the routine use of such formula. ${ }^{5,12}$

The ESPGHAN committee on nutrition has concluded that at present, there are insufficient data to recommend the routine use of probiotic- and/or prebiotic-supplemented formula. Yet the administration of probiotic (single or in combination) supplementation to infant or follow-on formula, and given beyond early infancy, may be associated with some clinical benefits. These include a reduction in the risk of nonspecific gastrointestinal infections, a reduced risk of antibiotic use and a lower frequency of colic or irritability. Reviewed studies have varied with regard to methodological quality, the specific strains studied, the duration of the interventions and the doses used.

The FAO supports the use of prebiotics in infant formula for infants aged five months and older, as they have a more mature immune system. Products containing prebiotics or probiotics are not recommended for immunocompromised infants, ill preterm neonates and children with indwelling medical devices. ${ }^{5}$

\section{Conflict of interest}

The authors declare that they have no financial or personal relationships which may have inappropriately influenced them in writing this paper.

\section{References}

1. Agostoni C, Axelsson I, Braegger C, et al. Probiotic bacteria in dietetic products for infants: a commentary by the ESPGHAN Committee on Nutrition. J Pediatr Gastroenterol Nutr. 2004;38(4):365-374.

2. Boehm G, Moro G. Structural and functional aspects of prebiotics used in infant nutrition. J Nutr. 2008;138(9):1818S-1828S.

3. Veereman-Wauters $\mathrm{G}$. Application of prebiotics in infant foods. $\mathrm{Br} \mathrm{J}$ Nutr. 2005;93(Suppl 1):S57-S60

4. Parracho $\mathrm{H}, \mathrm{McC}$ artney $\mathrm{AL}$, Gibson GR. Probiotics and prebiotics in infant nutrition. Proc Nutr Soc. 2007;66(3):405-411.

5. Thomas DW, Greer FR, American Academy of Pediatrics Committee on Nutrition, American Academy of Pediatrics Section on Gastroenterology, Hepatology, and Nutrition. Probiotics and prebiotics in pediatrics. Pediatrics. 2010;126(6):1217-1231.

6. Rao S, Srinivasjois R, Patole S. Prebiotic supplementation in full-term neonates: a systematic review of randomized controlled trials. Arch Pediatr Adolesc Med. 2009;163(8):755-764

7. Saavedra JM. Use of probiotics in pediatrics: rationale, mechanisms of action, and practical aspects. Nutr Clin Pract. 2007;22(3):351-365.

8. Srinivasjois R, Rao S, Patole S. Prebiotic supplementation of formula in preterm neonates: a systematic review and meta-analysis of randomised controlled trials. Clin Nutr. 2009;28(3):237-242.

9. Chouraqui J, Grathwohl D, Labaune J, et al. Assessment of the safety, tolerance, and protective effect against diarrhea of infant formulas containing mixtures of probiotics or probiotics and prebiotics in a randomized controlled trial. Am J Clin Nutr. 2008;87(5):1365-1373.

10. Saavedra JM, Abi-Hanna A, Moore N, Yolken RH. Long-term consumption of infant formulas containing live probiotic bacteria: tolerance and safety. Am J Clin Nutr. 2004;79(2):261-267.

11. Arslanoglu S, Moro GE, Boehm G. Early supplementation of prebiotic oligosaccharides protects formula-fed infants against infections during the first 6 months of life. J Nutr. 2007;137(11):2420-2424.

12. Braegger $\mathrm{C}$, Chmielewska A, Decsi $T$, et al. Supplementation of infant formula with probiotics and/or prebiotics: a systematic review and comment by the ESPGHAN committee on nutrition. J Pediatr Gastroenterol Nutr. 2011;52(2):238-250.

13. Szajewska H, Mrukowicz JZ. Probiotics in the treatment and prevention of acute infectious diarrhea in infants and children: a systematic review of published randomized, double-blind, placebo-controlled trials. J Pediatr Gastroenterol Nutr. 2001;33(Suppl 2):S17-S25.

14. Van Niel CW, Feudtner C, Garrison MM, Christakis DA. Lactobacillus therapy for acute infectious diarrhea in children: a meta-analysis. Pediatrics. 2002;109(4):678-684

15. Huang JS, Bousvaros A, Lee JW, et al. Efficacy of probiotic use in acute diarrhea in children: a meta-analysis. Dig Dis Sci. 2002;47(11):2625-2634.

16. Allen SJ, Okoko B, Martinez E, et al. Probiotics for treating infectious diarrhoea. [Cochrane review]. In The Cochrane Library, Issue 2, 2004. Oxford: Update Software.

17 Szajewska H, Ruszczynski M, Radzikowski A. Probiotics in the prevention of antibiotic-associated diarrhea in children: a meta-analysis of randomized controlled trials. J Pediatr. 2006;149(3):367-372.

18. Osborn DA, Sinn JK. Probiotics in infants for prevention of allergic disease and food hypersensitivity. [Cochrane review]. In The Cochrane Library, Issue 4, 2007. Oxford: Update Software.

19. Osborn DA, Sinn JK. Prebiotics in infants for prevention of allergic disease and food hypersensitivity. [Cochrane review]. In The Cochrane Library, Issue 4, 2007. Oxford: Update Software. 